\title{
A DYNAMIC RECONSTRUCTION APPROACH FOR CEREBRAL BLOOD FLOW QUANTIFICATION WITH AN INTERVENTIONAL C-ARM CT
}

\author{
A. Fieselmann ${ }^{1,2,3}$, A. Ganguly ${ }^{4}$, Y. Deuerling-Zheng ${ }^{3}$, M. Zellerhoff ${ }^{3}$, J. Boese ${ }^{3}$, J. Hornegger ${ }^{1,2}$, \\ R. Fahrig ${ }^{4}$ \\ ${ }^{1}$ Pattern Recognition Lab, University Erlangen-Nuremberg, Erlangen, Germany \\ ${ }^{2}$ School in Advanced Optical Technologies (SAOT), Erlangen, Germany \\ ${ }^{3}$ Siemens AG, Healthcare Sector, Forchheim, Germany \\ ${ }^{4}$ School of Medicine, Stanford University, Stanford, CA
}

\begin{abstract}
Tomographic perfusion imaging is a well accepted method for stroke diagnosis that is available with current CT and MRI scanners. A challenging new method, which is currently not available, is perfusion imaging with an interventional $\mathrm{C}$-arm CT that can acquire 4-D images using a C-arm angiography system. This method may help to optimize the workflow during catheter-guided stroke treatment. The main challenge in perfusion $\mathrm{C}$-arm $\mathrm{CT}$ is the comparably slow rotational speed of the C-arm (approximately 5 seconds) which decreases the overall temporal resolution. In this work we present a dynamic reconstruction approach optimized for perfusion C-arm CT based on temporal estimation of partially backprojected volumes. We use numerical simulations to validate the algorithm: For a typical configuration the relative error in estimated arterial peak enhancement decreases from $14.6 \%$ to $10.5 \%$ using the dynamic reconstruction. Furthermore we present initial results obtained with a clinical C-arm CT in a pig model.
\end{abstract}

\section{INTRODUCTION}

A stroke is a serious medical emergency that requires fast diagnosis and treatment. The common imaging modalities for the diagnosis of acute stroke are perfusion CT and perfusion MRI which quantify the regional blood flow and volume in the brain tissue. The major aim is to identify tissue regions that are salvagable and may profit from stroke therapy.

The time window after stroke onset for a successful therapy is very narrow. Intra-arterial thrombolysis has a larger time window than conventional intra-venous thrombolysis but needs to be performed in the interventional suite. With CTlike imaging on a C-arm angiography system (C-arm CT [1]) it is possible to aquire large volume tomographic images during an intervention. Recently it has been shown that using a dedicated scan and injection protocol cerebral blood volume (CBV) can be quantified with C-arm CT [2]. Cerebral blood flow $(\mathrm{CBF})$ is a parameter that is measured by analyzing a sequence of images aquired at short temporal intervals after the injection of a contrast bolus. Due to the long $\mathrm{C}$-arm rotation time it is challenging to measure $\mathrm{CBF}$ with current $\mathrm{C}$-arm $\mathrm{CT}$ scanners.

Neukirchen et al. [3] have proposed a dynamic iterative reconstruction algorithm to compensate the effects of the slow $\mathrm{C}$-arm rotational speed. A dynamic reconstruction approach for conventional CT scanners based on filtered backprojection was presented by Montes et al. [4]. Their approach utilizes a slowly rotating scanner in order to reduce X-ray dose.

In this paper we present our dynamic reconstruction approach for $\mathrm{CBF}$ measurements. It is adapted to the scan properties of slowly-rotating $\mathrm{C}$-arm CT scanners. We use numerical simulations to investigate the algorithm under different scan conditions. Finally, we present first results from $\mathrm{CBF}$ measurements with a clinical $\mathrm{C}$-arm $\mathrm{CT}$ in a pig model.

\section{MATERIALS}

\subsection{Dynamic Reconstruction by Temporal Estimation of Partially Backprojected Volumes}

Standard cone-beam reconstruction algorithms require constant attenuation coefficients of the object during the acquisition. This requirement is not fulfilled in perfusion imaging due to the temporal change of contrast concentration. However, in perfusion CT the scan times are sufficiently short to assume constant attenuation coefficients during one acquisition. Current interventional C-arm CT scanners have acquisition times of several seconds and this assumption is not valid. Inconsistent data sets (where attenuation coefficients vary during the acquisition) can generate artifacts when using standard reconstruction algorithms [5].

In a typical C-arm $\mathrm{CT}$ system the $\mathrm{C}$-arm rotates in alternating directions (rotation time $T_{\text {rot }}$ ) with the view angle in the interval $\left[0, \alpha_{\max }\right]$. A time interval $T_{\mathrm{w}}$ is needed between two successive rotations. The period $T_{p}$ is the time between the starts of two same-directional rotations (Fig. 1).

Given $N_{\text {rot }}$ rotations of the C-arm we acquire for each 


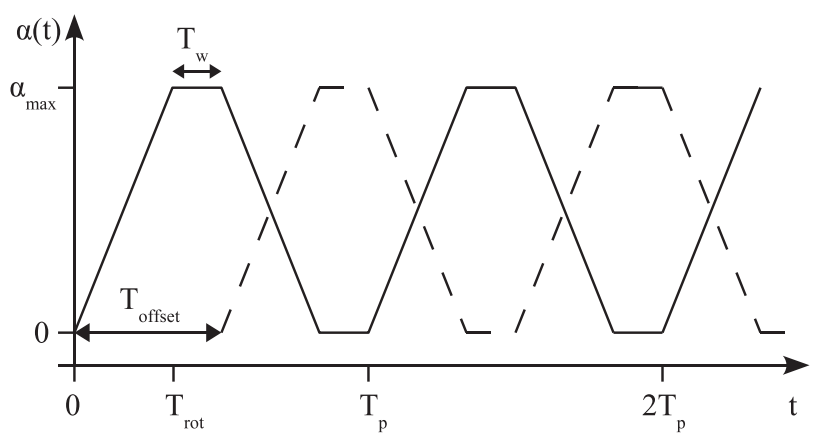

Fig. 1. Plot of the view angle $\alpha(t)$ of a C-arm CT scanner against time $t$. Overall temporal sampling in perfusion imaging can be increased by adding a second multi-rotational sequence (dashed line).

view angle $\alpha$ a sequence of cone-beam projections

$$
P_{\alpha}(\gamma, \phi, k)=P_{\alpha}\left(\gamma, \phi, t_{\alpha}(k)\right)
$$

(fan-angle $\gamma$, cone-angle $\phi$ ) at time points $t_{\alpha}(k)$ with $1 \leq k \leq$ $N_{\text {rot }}$. To reconstruct a volume at time $t$ we can approximate a consistent data set by temporal interpolation (with interpolation functions $\varphi_{\alpha, k}(t)$ ) in projection space, assuming that the contrast-induced temporal enhancement function is sufficiently smooth.

$$
P_{\alpha}(\gamma, \phi, t) \approx \sum_{k=1}^{N_{\text {rot }}} P_{\alpha}(\gamma, \phi, k) \cdot \varphi_{\alpha, k}\left(t-t_{\alpha}(k)\right)
$$

In C-arm CT scanners the projections may not be acquired at exactly the same angular position in forward and reverse rotations. To take this into account and to increase computational speed the interpolation can also be done in partially backprojected volumes. We define the operator $\operatorname{PFBP}_{\alpha_{1}}^{\alpha_{2}}(\mathbf{x})\left\{P_{\alpha}\right\}$ for (partial) filtered backprojection at position $\mathbf{x}$ using projections $P_{\alpha}$ with $\alpha \in\left[\alpha_{1}, \alpha_{2}\right]$. For the filtered backprojection a short-scan Feldkamp-type algorithm [6] can be used. A normal reconstruction at $\mathbf{x}$ is given by the sum of all partial filtered backprojections

$$
\mu(\mathbf{x})=\sum_{j=0}^{M-1} \operatorname{PFBP}_{j \cdot \Delta \alpha}^{(j+1) \cdot \Delta \alpha}(\mathbf{x})\left\{P_{\alpha}\right\}
$$

with $\Delta \alpha=\alpha_{\max } / M$ where $M$ is the number of angular intervals. In general, the angular intervals $\Delta \alpha$ of the partial backprojections can also be non-uniform. Dynamic reconstruction by temporal estimation of partially backprojected volumes is given by

$$
\begin{array}{r}
\mu(\mathbf{x}, t)=\sum_{j=0}^{M-1} \sum_{k=1}^{N_{\text {rot }}} \operatorname{PFBP}_{j \cdot \Delta \alpha}^{(j+1) \cdot \Delta \alpha}(\mathbf{x})\left\{P_{\alpha}(k)\right\} \ldots \\
\cdot \varphi(j+0.5) \cdot \Delta \alpha, k\left(t-t_{(j+0.5) \cdot \Delta \alpha}(k)\right)
\end{array}
$$

Montes et al. [4] have used a similar approach for dynamic reconstruction of data acquired in the normal CT scan geometry. Our extension for C-arm CT scanners uses interpolation functions $\varphi_{\alpha, k}(t)$ for irregular sampled data. They can be implemented e.g. as linear or cubic splines functions which are computationally fast. Other interpolation methods for nonuniformly sampled data are available but may be computationally more expensive [7].

In order to increase the temporal sampling of projection space an interleaved scanning protocol with $N_{\text {seq }}$ multirotational sequences can be used (Fig. 1 shows an example with two sequences). A new bolus with identical parameters is injected for each sequence. The temporal offset between the start of the sequences (normalized to the time of injection) can be given as $T_{\text {offset }}=T_{p} / N_{\text {seq }}$. The data from this scanning scheme is used as input to the dynamic reconstruction in (4) where the interpolation then uses the data from different sequences to estimate new partially backprojected volumes.

\subsection{Cerebral Blood Flow Quantification}

The indicator-dilution theory [8] relates a concentration time curve $c_{t}(t)$ in tissue and a time curve $c_{a}(t)$ in a reference artery by

$$
c_{t}(t)=\mathrm{CBF} \cdot \int_{0}^{t} c_{a}(\tau) r(t-\tau) d \tau
$$

where the residue function $r(t)$ depends on the distribution of transit times in tissue. The product $(\mathrm{CBF} \cdot r(t))$ can be computed by numerical deconvolution using e.g. the TSVD algorithm [9]. The maximum of $r(t)$ is one and therefore $\mathrm{CBF}$ can be computed as the maximum of $(\mathrm{CBF} \cdot r(t))$.

\subsection{Numerical Simulations}

We validated our dynamic reconstruction algorithm using different attenuation time curves. The arterial attenuation time curve $c_{a}(t)$ was modeled as a gamma-variate function [9] with peak enhancement $A$ and shape parameters $a$ and $b$.

$$
c_{a}(t)=\frac{A \cdot\left(t-t_{0}\right)^{a}}{\left(a \cdot b \cdot \mathrm{e}^{-1}\right)^{a}} \cdot \mathrm{e}^{-\left(t-t_{0}\right) / b} \cdot u\left(t-t_{0}\right)
$$

$u(t)$ is the step function and $t_{0}$ denotes the time of arrival of the contrast agent in the vessel. We chose literature values of $a=3, b=1.5$ [9] and set $A=100$. The tissue attenuation time curves were computed using (5) with an exponentially decaying residue function

$$
r(t)=\mathrm{e}^{-t /(\mathrm{CBV} / \mathrm{CBF})}
$$

where $\mathrm{CBV}=4 \mathrm{ml} / 100 \mathrm{~g}$ and $\mathrm{CBF}=[20,60] \mathrm{ml} / 100 \mathrm{~g} / \mathrm{min}$ to represent ischemic and healthy tissue respectively.

We investigated the reconstruction of the peak enhancement in the artery and in tissue as a function of the rotation time $T_{\text {rot }}$ and set $T_{w}=1 \mathrm{~s}$. Each simulation is repeated 10 
times with different scan start times relative to the start of the attenuation time curves. For the forward projection and reconstruction we took a parallel-beam geometry $\left(\alpha_{\max }=\pi\right)$ with a vessel centered in the origin. We used a cubic spline as interpolation function $\varphi_{\alpha, k}(t)$. We also evaluated the error

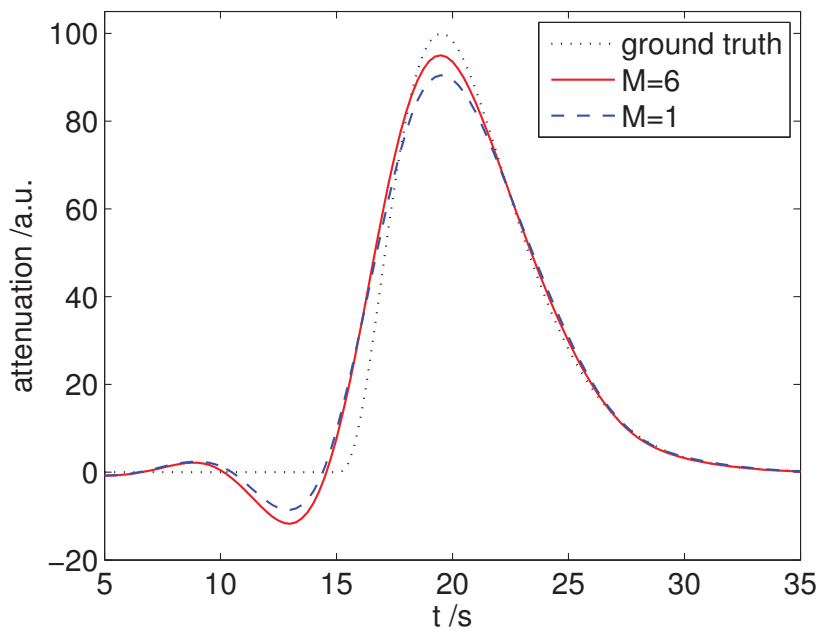

Fig. 2. Reconstruction of a typical arterial time attenuation curve using two different numbers $M$ of angular intervals.

in the peak enhancement value as a function of the different numbers $M$ of angular intervals under the same conditions.

\subsection{Experimental Investigation in a Pig Model}

We investigated CBF imaging with a clinical C-arm CT (Axiom Artis $d$ TA with DynaCT, Siemens AG) in one healthy pig. The rotation time $T_{\text {rot }}$ was $4.3 \mathrm{~s}$ followed by $1.25 \mathrm{~s}$ wait time $T_{w}$. We injected a bolus with $50 \%$ contrast concentration at $6 \mathrm{ml} / \mathrm{s}$ during $8 \mathrm{~s}$ into the aortic arch using a pig-tail catheter. We used 2 interleaved scans $\left(T_{\text {offset }}=5.55 s\right)$ and set $M=6$ for the reconstruction. Quantitative evaluation of CBF maps was done with perfusion CT of the same pig with same injection profiles as a gold standard. After registration of the $\mathrm{C}$-arm CT and CT data, mean CBF values were measured in 6 circular (radius $2 \mathrm{~mm}$ ) regions of interest (ROI) per slice.

\section{RESULTS}

A typical reconstruction (scanner settings $T_{\text {rot }}=3 \mathrm{~s}$ ) of the simulated arterial attenuation time curve at $0.25 \mathrm{~s}$ resolution of the interpolation is shown in Fig. 2. The peak is underestimated for both values of $M$. The underestimation is obviously higher for $M=1$ than for $M=6$. The results for the reconstructed peak enhancement depending on different numbers $M$ is shown in Fig. $3\left(T_{\text {rot }}=3 s\right)$. The reconstructed peak enhancement values are closer to the ground truth value for $M>1$ than $M=1$. The standard deviation increases for $M>1$.

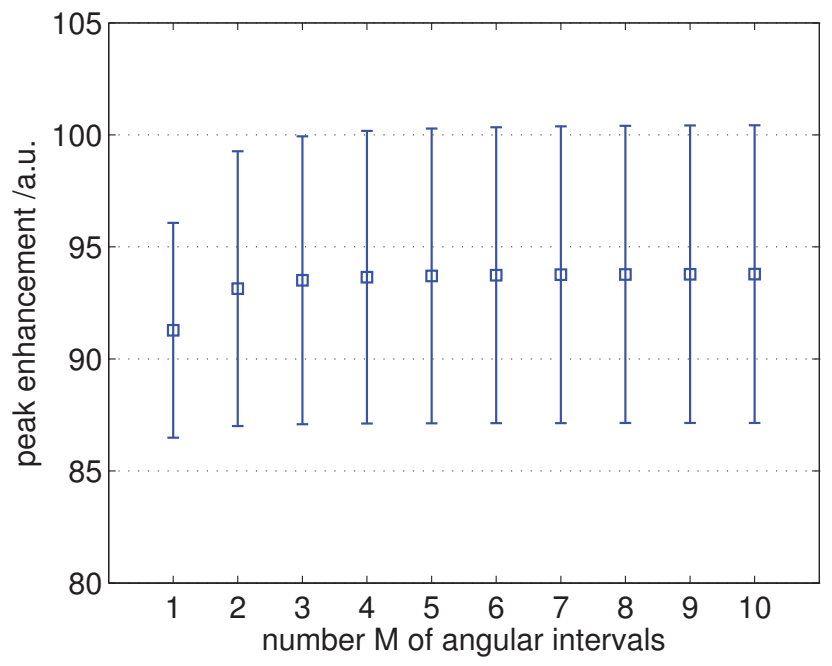

Fig. 3. Reconstructed arterial peak enhancement (ground truth value is 100) for different numbers $M$ of angular interpolation intervals. Mean and standard deviations are shown for simulations that were repeated for different scans start times.

The dependency of the reconstructed peak enhancement values on the rotation time $T_{\text {rot }}$ are shown in Fig. 4 . The peaks are systematically underestimated and the peak values decrease with increasing $T_{\text {rot }}$. The underestimation is higher in the arterial time attenuation curve than in the tissue time attenuation curve. The relative error in the arterial peak estimation decreases from $14.6 \%(M=1)$ to $10.5 \%(M=6)$ for $T_{\text {rot }}=4 s$.

Fig. 5 shows the CBF map from the measurement in the pig model. The mean $\mathrm{CBF}$ values in the ROIs tend to be overestimated using C-arm CT compared to CT. The Pearson $r$ coefficient that is a measure for the correlation between the mean CBF in the ROIs in C-arm CT and CT is 0.84 $(\mathrm{p}<0.001)$.

\section{DISCUSSION AND CONCLUSION}

The results in section 3 show that the reconstructed peak enhancement values of the attenuation time curves are systematically underestimated. The convolution in (5) smoothes the tissue time curves such that the interpolation is more reliable for the tissue than for the arterial time curves which contain more high-frequency components. If the relative underestimation of the peak value of the reference artery is greater than the relative underestimation of the peak value of the tissue time curve then (according to (5)) the CBF value is overestimated. This can explain the overestimation of $\mathrm{CBF}$ values in the measurements in the pig model using C-arm CT (as compared to the gold standard CT). For $M=1$ the dynamic reconstruction approach reduces to a standard reconstructi- 


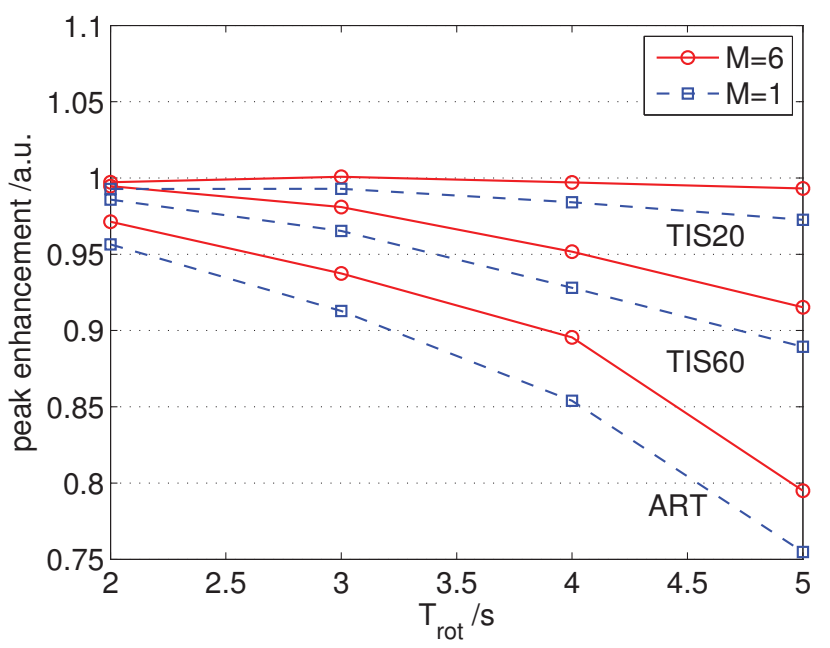

Fig. 4. Reconstructed peak enhancement of typical time attenuation curves using a simulated $\mathrm{C}$-arm CT scanner (ART: artery, [TIS20 TIS60]: tissue with blood flow of [20 60] $\mathrm{ml} / 100 \mathrm{~g} / \mathrm{min}$ ). The values are normalized with the ground truth. $M$ is the number of angular intervals used for interpolation.

on with subsequent temporal interpolation of the time curve. This standard reconstruction can be interpreted as a sampling of the input signal convolved with a rectangular function (i.e. a filtering with a low-pass filter). The peak value reconstruction are significantly closer to the ground truth values using the dynamic reconstruction approach $(M>1)$ than a standard reconstruction approach $(M=1)$. The standard deviation values in Fig. 3 show that the times of the scan start relative to the perfusion signal have a major impact on the peak enhancement reconstruction value.

This works shows that it is possible to reconstruct typical perfusion time attenuation curves using the scan geometry of $\mathrm{C}$-arm CT scanners. However the reconstruction accuracy de-

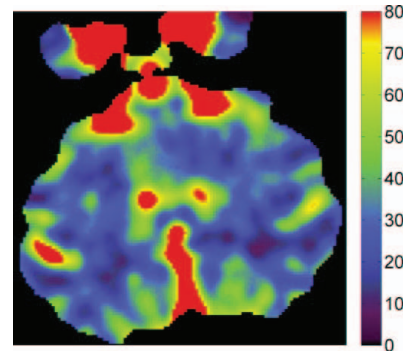

(a) C-arm CT

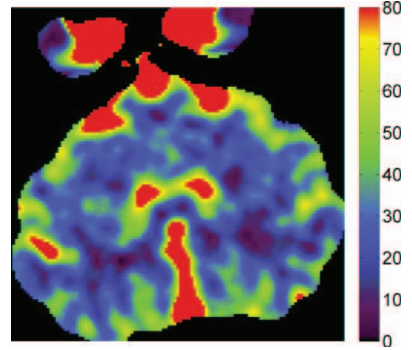

(b) $\mathrm{CT}$
Fig. 5. CBF parameter maps (unit $\mathrm{ml} / 100 \mathrm{~g} / \mathrm{min}$ ) measured in a healthy pig model using perfusion $\mathrm{C}$-arm $\mathrm{CT}$ with the dynamic reconstruction approach and perfusion $\mathrm{CT}$. pends on the rotational speed of the C-arm and the temporal characteristic of the perfusion signals. An increase in rotational speed of the $\mathrm{C}$-arm and higher detector read-out rates in current C-arm CT scanners can improve the estimation of signal characteristics and $\mathrm{CBF}$ values.

\section{ACKNOWLEDGEMENTS}

The authors gratefully acknowledge funding of the Erlangen Graduate School in Advanced Optical Technologies (SAOT) by the German Research Foundation (DFG) in the framework of the German excellence initiative.

\section{REFERENCES}

[1] M. Zellerhoff, B. Scholz, E.-P. Rührnschopf, and T. Brunner, "Low contrast 3-D reconstruction from C-arm data," in Proc SPIE Medical Imaging, 2005, vol. 5745, pp. 646655.

[2] A.S. Ahmed, M. Zellerhoff, C.M. Strother, K.A. Pulfer, T. Redel, Y. Deuerling-Zheng, K. Royalty, D. Consigny, and D.B. Niemann, "C-arm CT measurement of cerebral blood volume: An experimental study in canines," Am J Neuroradiol, vol. 30, no. 5, pp. 917-922, 2009.

[3] C. Neukirchen and S. Hohmann, "An iterative approach for model-based tomographic perfusion estimation," in Proc Fully 3-D, 2007, pp. 104-107.

[4] Pau Montes and Günter Lauritsch, "A temporal interpolation approach for dynamic reconstruction in perfusion CT," Med Phys, vol. 34, no. 7, pp. 3077-3092, Jul 2007.

[5] S. Bonnet, A. Koenig, S. Roux, P. Hugonnard, R. Guillemaud, and P. Grangeat, "Dynamic X-ray computed tomography," Proc IEEE, vol. 91, no. 10, pp. 1574-1587, 2003.

[6] L. A. Feldkamp, L. C. Davis, and J. W. Kress, "Practical cone-beam algorithm," J Opt Soc Am, vol. A1, pp. 612$619,1984$.

[7] W. H. Press, S. A. Teukolsky, W. T. Vetterling, and B. P. Flannery, Numerical Recipes, Cambridge University Press, 3rd edition, 2007.

[8] P. Meier and K.L. Zierler, "On the theory of the indicatordilution method for measurement of blood flow and volume," Appl Physiol, vol. 6, pp. 731-744, 1954.

[9] L. Østergaard, R. M. Weisskoff, D. A. Chesler, C. Gyldensted, and B. R. Rosen, "High resolution measurement of cerebral blood flow using intravascular tracer bolus passages. part I: Mathematical approach and statistical analysis," Magn Reson Med, vol. 36, no. 5, pp. 715-725, 1996. 